\title{
Better-Than-Worst-Case Design Methodology for a Compact Integrated Switched-Capacitor DC-DC Converter
}

\author{
Dongkwun Kim \\ Electrical Engineering \\ Columbia University, New York, NY, USA \\ dk2990@columbia.edu
}

\author{
Mingoo Seok \\ Electrical Engineering \\ Columbia University, New York, NY, USA \\ ms4415@columbia.edu
}

\begin{abstract}
We suggest a new methodology in co-designing an integrated switched-capacitor converter and a digital load. Conventionally, a load has been specified to the minimum supply voltage and the maximum power dissipation, each found at her own worst-case process, workload, and environment condition. Furthermore, in designing an SC DC-DC converter toward this worst-case load specification, designers often have been adding another separate pessimistic assumption on power-switch's resistance and flyingcapacitor's density of an SC converter. Such worst-case design methodology can lead to a significantly over-sized flying capacitor and thereby limit on-chip integration of a converter. Our proposed methodology instead adopts the better than worst-case (BTWC) perspective to avoid over-design and thus optimizes the area of an SC converter. Specifically, we propose BTWC load modeling where we specify non-pessimistic sets of supply voltage requirement and load power dissipation across variations. In addition, by considering coupled variations between the SC converter and the load integrated in the same die, our methodology can further reduce the pessimism in power-switch's resistance and capacitor density. The proposed co-design methodology is verified with a $2: 1 \mathrm{SC}$ converter and a digital load in a $65 \mathrm{~nm}$. The resulted converter achieves more than one order of magnitude reduction in the flying capacitor size as compared to the conventional worstcase design while maintaining the target conversion efficiency and target throughput. We also verified our methodology with a wide range of load characteristics in terms of their supply voltages and current draw and confirmed the similar benefits.
\end{abstract}

\section{CCS Concepts}

Hardware $\rightarrow$ Integrated circuits

\section{Keywords}

Fully integrated power converter, Switched-capacitor (SC) DC-DC converter, 2:1 DC-DC converter

\section{Introduction}

https://doi.org/10.1145/3218603.3218610In today's multicore processors, the per-core dynamic voltage frequency scaling (DVFS) is highly desirable to optimize energy-efficiency and

Permission to make digital or hard copies of all or part of this work for personal or classroom use is granted without fee provided that copies are not made or distributed for profit or commercial advantage and that copies bear this notice and the full citation on the first page. Copyrights for components of this work owned by others than ACM must be honored. Abstracting with credit is permitted. To copy otherwise, or republish, to post on servers or to redistribute to lists, requires prior specific permission and/or a fee.

Request permissions from Permissions@acm.org.

ISLPED '18, July 23-25, 2018, Seattle, WA, USA

(C) 2018 Association for Computing Machinery.

ACM ISBN 978-1-4503-5704-3/18/07 \$15.00

https://doi.org/10.1145/3218603.3218610 performance under the thermal and reliability envelop $[1,2]$. To implement the per-core DVFS, a key challenge is to enable compact and thus fully-integrated DC-DC converters. Board-level power conversion is increasingly less attractive as the number of cores in a processor rapidly increases. A switched-capacitor (SC) based converter is often more favorable than an inductor-based converter due to their higher power density and CMOS process compatibility [3]. Recent works show the promise to implement fully-integrated SC converters for a low-power digital load that operate at deeplyscaled supply voltage [2].

Conventionally, an SC converter and a load circuit are designed based on the worst-case design paradigm, i.e., design them such that they can achieve the promised performance in the worst-case process, voltage, temperature, and workload condition. In addition, even though they are integrated in the same chip, they are often designed rather separately without much consideration on the coupling between them.

Such conventional methodology starts typically by pessimistically specifying a load. The key parameters to specify for a load are: (i) the maximum current draw, and (ii) the minimum supply voltage $\left(V_{D D}\right)$. First, the $V_{D D}$ is specified such that it can achieve a target clock frequency even in the worst-case corner. In our exemplary case with a digital circuit whose critical path is 40 Fan-Out-of-4 (FO4) delays in a $65 \mathrm{~nm}$, we need at least $0.45 \mathrm{~V} \mathrm{VDD}$ to meet the target clock frequency $(80 \mathrm{MHz})$ at the $\mathrm{SS}$ and $-20^{\circ} \mathrm{C}$ corner. Then next, we specify the worst-case current draw across process and temperature corners. As shown in Fig 1, at the $0.45 \mathrm{~V} \mathrm{~V} \mathrm{VD} \mathrm{that} \mathrm{is}$ just specified, the maximum current draw is estimated $\sim 3.1 \mathrm{~mA}$ at the $\mathrm{FF}$ and $80^{\circ} \mathrm{C}$ corner. This is about $24.8 \times$ larger than the current draw of $0.13 \mathrm{~mA}$ at the $\mathrm{SS}$ and $-20^{\circ} \mathrm{C}$ corner.

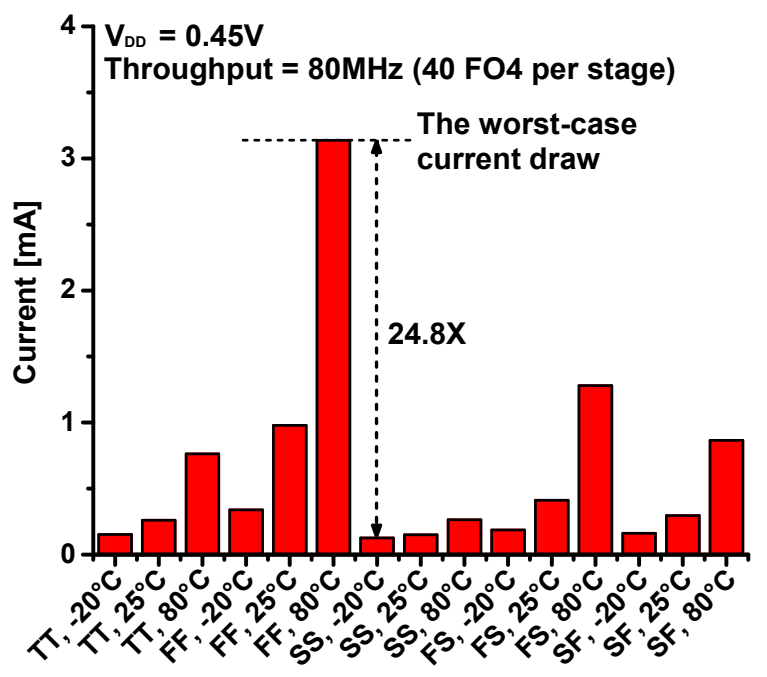

Fig. 1. The current draw of a near-threshold voltage digital circuit across process and temperature corners 
With this overly pessimistic load specification, the design of SC converters also becomes highly pessimistic. Furthermore, designers can add another separate pessimistic assumption on the resistance of power-switches and the capacitor density. Due to all of these overly pessimistic assumptions, the resulting flying capacitor, which typically dominates the size of an SC converter, can be significantly larger than necessary.

In this paper, to tackle this problem, we propose a methodology based on the better than worst case (BTWC) perspective [4]. First, we propose BTWC load specification where we aim to find nonpessimistic supply voltage requirements and load power dissipations. Next, we propose to consider coupled variations between a converter and a load in the design process. Since they are integrated in the same die, they share the similar variations. As a result, the proposed BTWC methodology can reduce the pessimism in sizing a flying capacitor and power transistors while maintaining target power conversion efficiency (PCE).

We experimented the proposed methodology with a 2:1 converter and a digital load circuit in a $65 \mathrm{~nm}$ CMOS. Our methodology can successfully reduce flying capacitor size by 4.1 to $42.9 \times$ over the conventional worst-case methodology while achieving the target computing throughput of the load as well as the target PCE of the converter. We also verified the proposed methodology across multiple load current levels, $\mathrm{V}_{\mathrm{DD}}$ regimes, and capacitor types, confirming the similar or better benefits.

The remaining paper is organized as follows. In Sec. 2, we will discuss the conventional worst-case design methodology for an integrated SC converter. In Sec. 3, we will outline our BTWC load specification. We will also discuss the BTWC converter design by considering the coupled variations between a load and a converter, together with our simulation results on the scaling of a flying capacitor. In Sec. 4, we will investigate the impact of load current, load $\mathrm{V}_{\mathrm{DD}}$, and on-chip capacitor type on the efficacy of our proposed methodology. Finally, we will conclude in Sec. 5.

\section{Conventional Worst-Case Methodology}

In this section, we describe the steps of the conventional worst-case design approach of an SC converter. For the integrated converter design, it is ideal to co-design a converter and a load. However, this quickly increases design complexity and simulation/verification time. Therefore, in many cases, a load is abstracted to a current source and a converter is designed toward satisfying the specifications of a load.

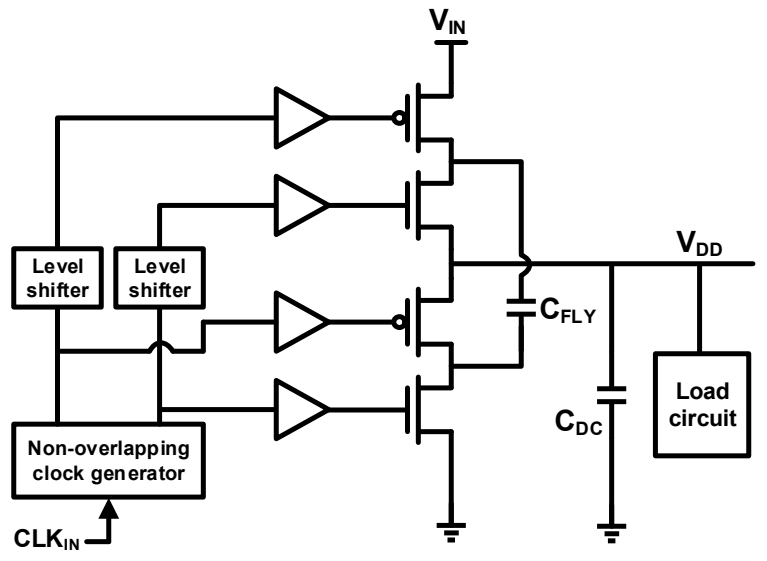

Fig. 2. Schematic of the benchmark circuits consisting of a 2:1 single-phase SC converter and an inverter-based load circuit

Fig. 2 shows a system of a converter and a load, which we use to benchmark the design methodologies in this paper. It is comprised

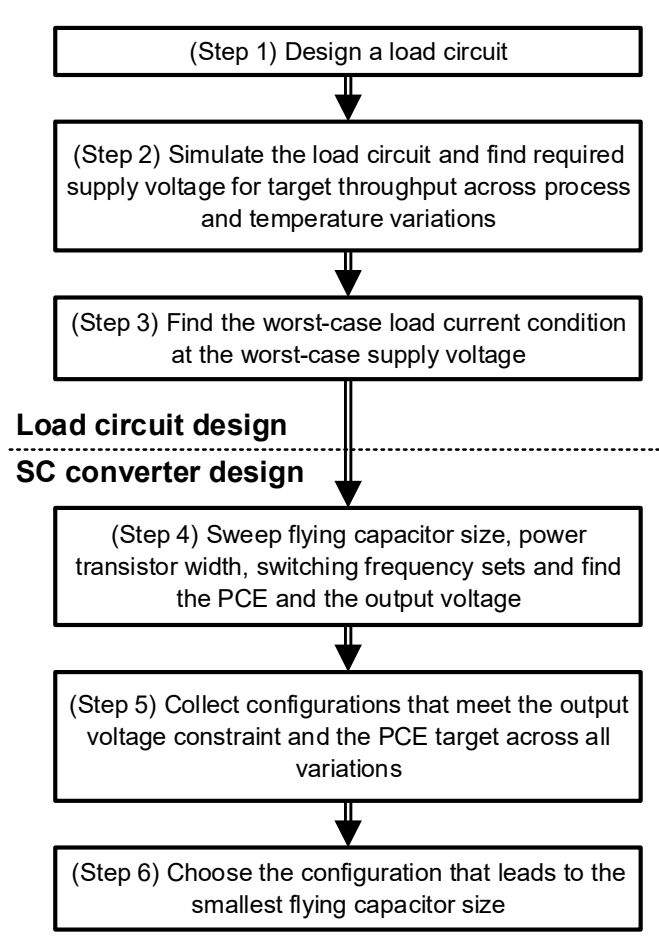

(a)

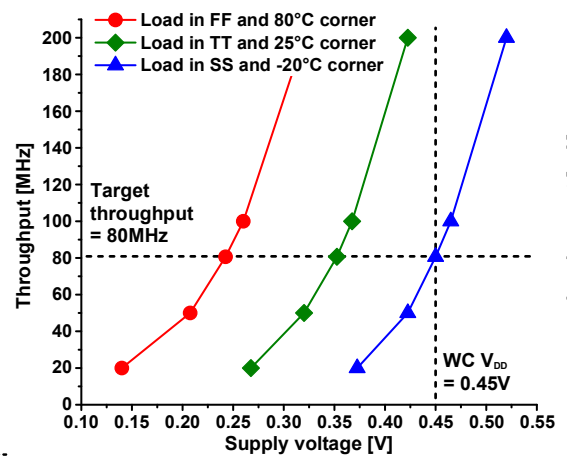

(b)

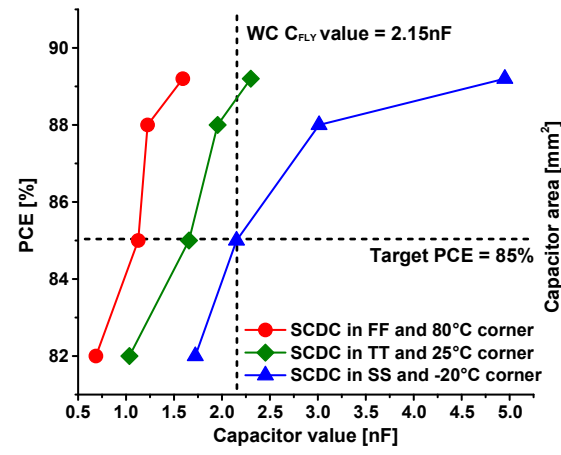

(d)

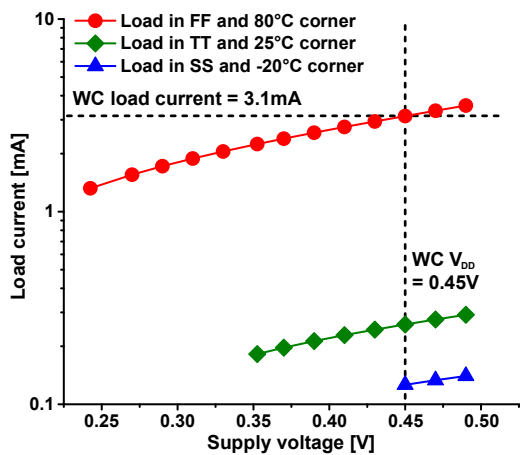

(c)

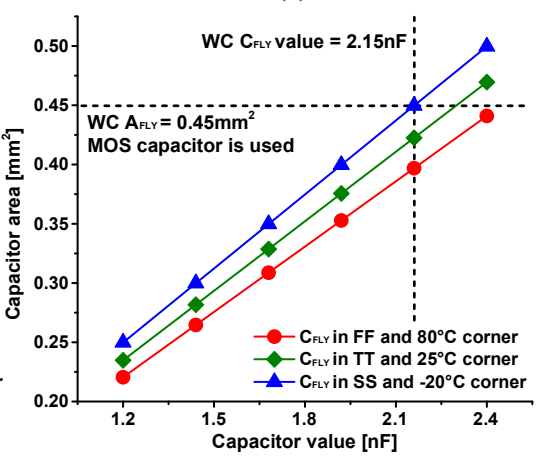

(e)

Fig. 3. (a) Conventional WC design methodology, (b) the minimum VDD for the target throughput, (c) the maximum ILD at the fixed minimum Vdd, (d) the minimum CFly for the target PCE, (e) the minimum Afly for the target CFLY 
of a single-phase 2:1 SC converter, an inverter-chain based load circuit, a non-overlapping clock generator, level shifters, buffers, and a decoupling capacitor $\left(\mathrm{C}_{\mathrm{DC}}\right)$. The load is 150 of $40 \mathrm{FO} 4$ inverter chains with nodal switching activity of 0.2 [5]. The area of the load circuits is estimated at $0.104 \mathrm{~mm}^{2}$ considering $80 \%$ cell utilization and the overhead of power rings and grids. The nonoverlapping clock generator is used to minimize crowbar current [6, 7]. Level shifters can reduce the swing of the converter clock by half, improving PCE [7]. All the capacitors are implemented in a MOS capacitor while other types of capacitors will be considered in Sec. 4.3. The power consumption of all the peripheral blocks is accounted for PCE calculation. The input voltage $\left(\mathrm{V}_{\mathrm{IN}}\right)$ is $1.0 \mathrm{~V}$.

As shown in Fig. 3(a), the first step of the worst-case methodology is to specify the load, namely finding the minimum $V_{D D}$ and the maximum current draw ( $\left.\mathrm{I}_{\mathrm{LD}}\right)$. We simulated the load across process and temperature corners and found $\mathrm{V}_{\mathrm{DD}}$ that achieves the target throughput of $80 \mathrm{MHz}$ across all the corners considered. As shown in Fig. 3(b), the worst-case $\mathrm{V}_{\mathrm{DD}}$ is found $0.45 \mathrm{~V}$ at the $\mathrm{SS}$ and $-20^{\circ} \mathrm{C}$ corner for the target throughput. We then set $\mathrm{V}_{\mathrm{DD}}$ to $0.45 \mathrm{~V}$ ( $\left.\mathrm{V}_{\mathrm{DD}}, \mathrm{WC}\right)$ and simulate the ILD of the load across process and temperature corners. As shown in Fig. 3(c), the worst-case ILD is found to be 3.1 $\mathrm{mA}$ at the $\mathrm{FF}$ and $80^{\circ} \mathrm{C}$ corner. Note that this is significantly larger than the $\mathrm{I}_{\mathrm{LD}}$ found at $\mathrm{TT}$ and $25^{\circ} \mathrm{C}$. Also, the minimum $\mathrm{V}_{\mathrm{DD}}$ and the maximum ILD are specified to two different corners, incurring overly pessimistic load specifications.

Next, a converter designer receives the load specifications found above (i.e., $\mathrm{V}_{\mathrm{DD}}>0.45 \mathrm{~V}, \mathrm{I}_{\mathrm{LD}}=3.1 \mathrm{~mA}$ ) and based on those starts to design a converter (the lower part of Fig. 3(a)). Specifically, we need to find the optimal sets of flying capacitor capacitance ( $\left.\mathrm{C}_{\mathrm{FLY}}\right)$, power transistor width $\left(\mathrm{W}_{\mathrm{P}}\right)$, and switching frequency $\left(\mathrm{F}_{\mathrm{S}}\right)$. To do this, we swept those parameters across process and temperature corners and collected the key performance metrics of an SC converter, namely the minimum output voltage (VouT,MIN) and PCE.

Based on the collected metrics, the designers now choose the configuration that meets system-level needs. In this benchmark, we choose the configuration that leads to the smallest $C_{F L Y}$ while making PCE greater than $85 \%$ and $\mathrm{V}_{\text {OUT,MIN }}$ greater than $\mathrm{V}_{\mathrm{DD} \text {,WC. }}$ We assume we can dynamically change $W_{P}$ and $F_{S}$ optimally to environmental changes. But we do not consider to modulate $\mathrm{C}_{\mathrm{FLY}}$ since the area of the flying capacitor is already committed to support the largest $\mathrm{C}_{\mathrm{FLY}}$ and the modulation cannot save its area. Fig. 3(d) shows the impact of $\mathrm{C}_{\mathrm{FLY}}$ on PCE across process and temperature corners. To achieve the target $\mathrm{PCE}$ at the worst-case corner ( $\mathrm{SS}$ and $-20^{\circ} \mathrm{C}$ ), we need $\mathrm{C}_{\mathrm{FLY}}>2.15 \mathrm{nF}$. Finally, we consider the worst-case capacitor density across process and temperature corners and determine the area of a flying capacitor (AfLY). As shown in Fig. 3(e), this another worst-case consideration leads to AfLY of $0.45 \mathrm{~mm}^{2}$. This AFLY is $\sim 4.3 \times$ larger than the size of the load $\left(0.104 \mathrm{~mm}^{2}\right)$.

\section{Proposed BTWC Methodology}

\subsection{Overview}

The worst-case methodology that we outlined in Sec. 2 has considered multiple pessimistic assumptions, e.g., the worst-case condition for the minimum $\mathrm{V}_{\mathrm{DD}}$, that for the maximum $\mathrm{I}_{\mathrm{LD}}$, that for the largest power-transistor resistance, and that for the smallest capacitor density. However, it is highly unlikely if not impossible that all the worst-case assumptions happen simultaneously. For example, an integrated converter would experience the similar process and temperature corner with the load. Therefore, it is not necessary to assume that they would be in two opposite corners.
To mitigate those pessimisms, therefore, we propose the BTWC design methodology for an integrated converter design. As shown in Fig. 4, our proposed methodology uses the BTWC specification instead of the worst-case specification for a load. Here we specify pairs of the minimum $V_{D D}$ and the maximum $I_{L D}$ in the same process and temperature corners. In addition, we consider that the converter and the load would experience the same process and temperature variations. With these less pessimistic assumptions, designers can determine $\mathrm{C}_{\mathrm{FLY}}, \mathrm{W}_{\mathrm{P}}$, and $\mathrm{F}_{\mathrm{S}}$ for the target PCE and the minimum AFLY.

\subsection{BTWC Load Specification}

We propose the BTWC load specification that reduces the pessimism in specifying the minimum $\mathrm{V}_{\mathrm{DD}}$ and the maximum ILD. As shown in Fig. 5(a), each process and temperature corner requires the different minimum $\mathrm{V}_{\mathrm{DD}}$. In near and sub-threshold voltage regime, the load becomes faster at the $\mathrm{FF}$ and $80^{\circ} \mathrm{C}$ corner. This is because the threshold voltage of a transistor $\left(\mathrm{V}_{\mathrm{TH}}\right)$ reduces at that corner, which exponentially increases weak-inversion drain current [8]. In our benchmark circuits (Fig. 2), the minimum $\mathrm{V}_{\text {DDS }}$ are $0.45 \mathrm{~V}$ at SS and $-20^{\circ} \mathrm{C}, 0.35 \mathrm{~V}$ at $\mathrm{TT}$ and $25^{\circ} \mathrm{C}$, and $0.24 \mathrm{~V}$ at $\mathrm{FF}$ and $80^{\circ} \mathrm{C}$.

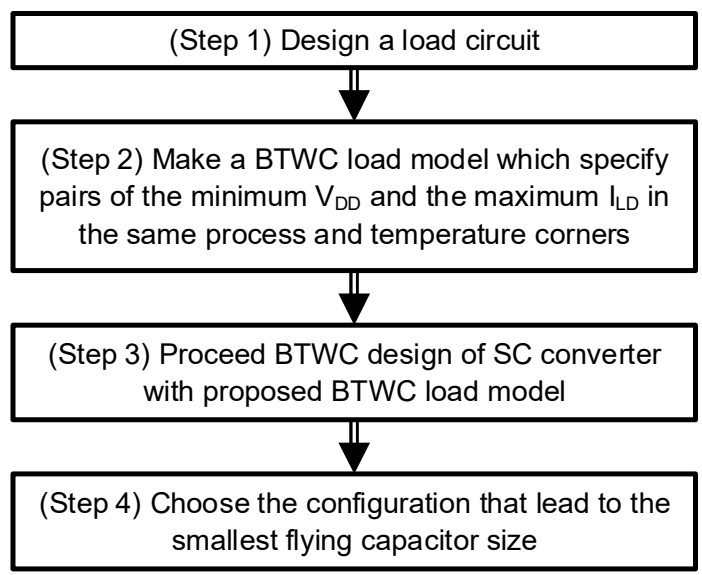

Fig. 4. Proposed BTWC methodology for an integrated SC converter

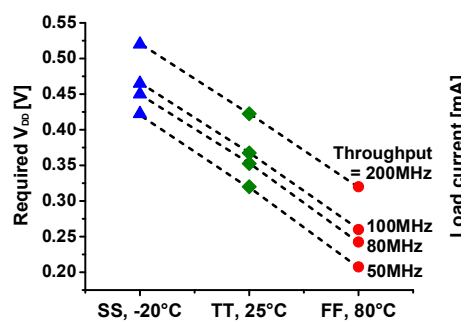

(a)

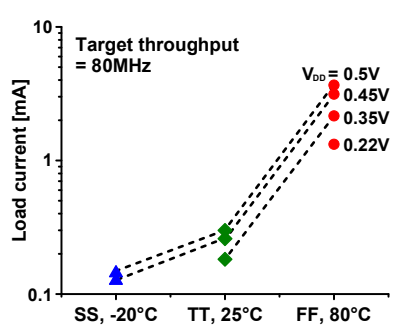

(b)
Fig. 5. (a) Minimum $V_{\text {DD }}$ for across different throughputs, (b) maximum $I_{L D}$ across $V_{\text {DDS }}$

In specifying the maximum ILD, we can consider this minimum VDD specification of each process and temperature corner, instead of considering a fixed $V_{D D}$ across all corners. For example, we can achieve the target throughput at lower $\mathrm{VDD}_{\mathrm{DD}}$ in the $\mathrm{FF}$ and $80^{\circ} \mathrm{C}$ corner. Therefore, the ILD at that corner, which used to be very high (24.8 $\times$ greater than the smallest ILD) in the experiment using the fixed VDD, can be reduced. As shown in Fig. 6, our experiment with the benchmark circuit (Fig. 2), the largest $I_{L D}$ is reduced to $1.32 \mathrm{~mA}$, which is $2.4 \times$ smaller than the previous worst-case specification of ILD, 3.1mA (Fig. 3). 


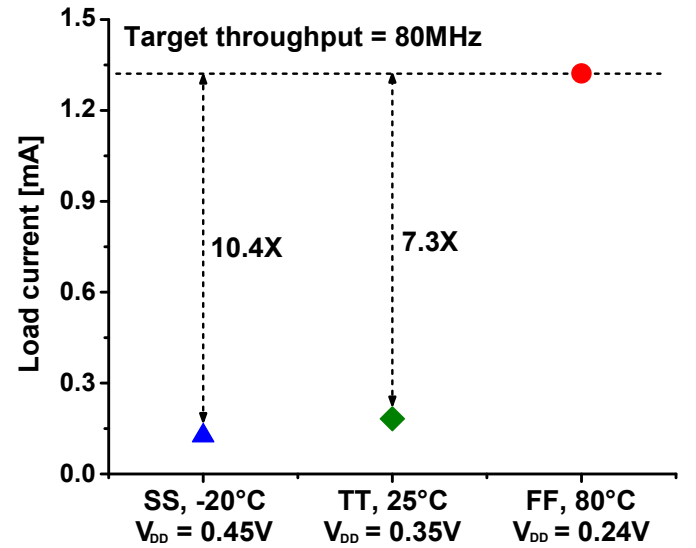

Fig. 6. Maximum load current draw at the $V_{D D}$ that is set for the target throughput at each corner

\subsection{BTWC Converter Design}

Using the BTWC load specification, we can design an integrated $\mathrm{SC}$ converter. Here, it is also desirable to mitigate the pessimistic assumptions on power switch resistance and capacitor density, which together determines the output impedance of an SC converter. Specifically, power transistor resistance creates the fast switching limit impedance $\left(\mathrm{R}_{\mathrm{FSL}}\right)$ i.e., the resistance impedes the current flow to and from a flying capacitor. The capacitor forms the other limit, called slow switching limit impedance (RssL), i.e., the capacitor size (together with other factors) affects the amount of charge transfer via charge sharing. These two form the asymptotic limits in the output impedance of an SC converter [3,9].
In the BTWC converter design, we consider that a converter and a load are integrated in the same die and thus share the similar process and temperature variations. This provides an important opportunity. In Sec. 3.2, we found that the maximum ILD is 1.32 $\mathrm{mA}$ at the $\mathrm{FF}$ and $80^{\circ} \mathrm{C}$ corner. This large $\mathrm{I}_{\mathrm{LD}}$ could require large CFLY. However, the converter is also in the same corner (FF and $80^{\circ} \mathrm{C}$ ). Thus, its RFsL is substantially reduced, allowing the converter to use faster Fs while using smaller CFLY to support the large ILD without PCE degradation.

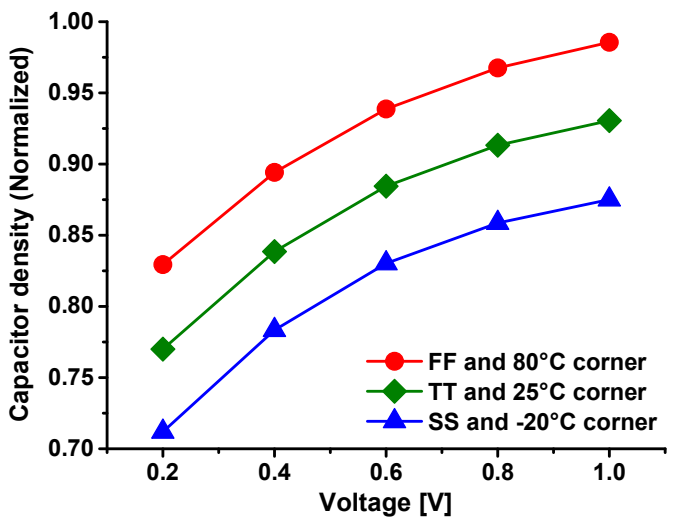

Fig. 7. Normalized MOS capacitor density across process, voltage, and temperature variations

Another opportunity is the capacitor density. In the $\mathrm{FF}$ and $80^{\circ} \mathrm{C}$ corner, where the converter needs the largest $\mathrm{C}_{\mathrm{FLY}}$, the MOS capacitor density is also larger than the other corner. This is because the $\mathrm{V}_{\mathrm{TH}}$ of MOS capacitor reduces, which inverts the channel more

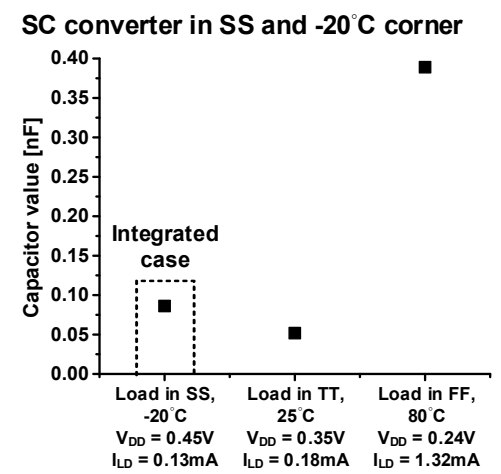

(a)

$\mathrm{SC}$ converter in $\mathrm{SS}$ and $-20^{\circ} \mathrm{C}$ corner

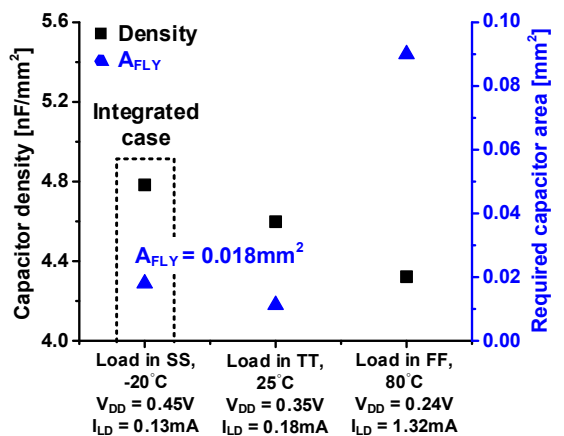

(d)

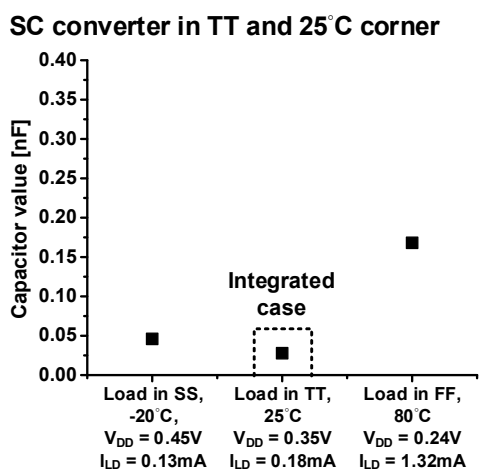

(b)

SC converter in TT and $25^{\circ} \mathrm{C}$ corner

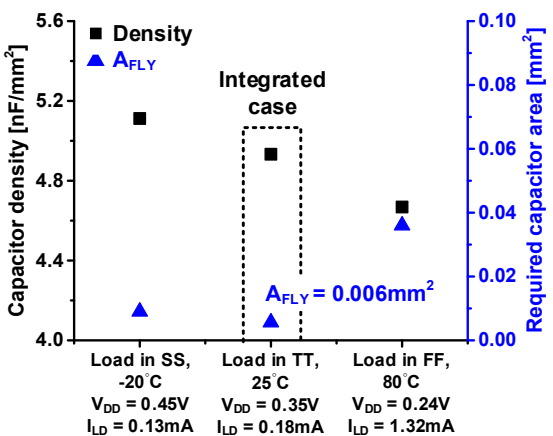

(e)

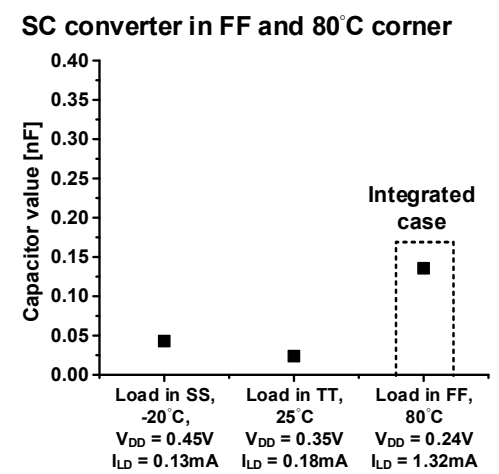

(c)

$\mathrm{SC}$ converter in $\mathrm{FF}$ and $80^{\circ} \mathrm{C}$ corner

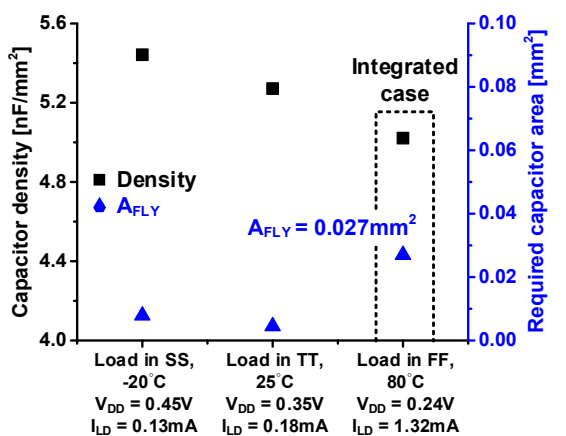

(f)

Fig. 8. The minimum $\mathrm{C}_{\mathrm{FLY}}$ for the converter in the (a) $\mathrm{SS}$ and $-20^{\circ} \mathrm{C}$, (b) $\mathrm{TT}$ and $25^{\circ} \mathrm{C}$, and (c) $\mathrm{FF}$ and $80^{\circ} \mathrm{C}$ corner. And, the $\mathrm{MOS}$ capacitor density and the minimum $A_{F L Y}$ in the (d) $\mathrm{SS}$ and $-20^{\circ} \mathrm{C}$, (e) TT and $25^{\circ} \mathrm{C}$, and (f) $\mathrm{FF}$ and $80^{\circ} \mathrm{C}$ corner. 
strongly [10]. The other factor that needs to be considered in capacitor density is that it is also a function of voltage. The MOS capacitor density reduces at lower $\mathrm{V}_{\mathrm{DD}}$ due to the less strong channel formation. The $\mathrm{FF}$ and $80^{\circ} \mathrm{C}$ corner have the smallest $\mathrm{V}_{\mathrm{DD}}$ requirement, reducing the density. Fig. 7 shows the variation of MOS capacitor density as a function of process, voltage, and temperature. Overall, we find a moderate amount of scaling in AFLY for the same $\mathrm{C}_{\mathrm{FLY}}$ requirement by considering the capacitor density variations.

Fig. 8 shows the simulation results of our experiment, i.e., designing the converter using the proposed BTWC methodology. Our experiment is based on the circuits shown in Fig. 2 in a $65 \mathrm{~nm}$, same as that in Sec. 2. First, we find required $\mathrm{C}_{\mathrm{FLY}}, \mathrm{W}_{\mathrm{P}}$, and $\mathrm{F}_{\mathrm{S}}$ that can support the BTWC load specification at each corner and achieve the target PCE. Fig. 8(a) shows the $\mathrm{C}_{\text {FLY }}$ requirement if the converter is at the $\mathrm{SS}$ and $-20^{\circ} \mathrm{C}$ corner. For the integrated converter design, only the leftmost data-point is valid because the load should be in the same SS and $-20^{\circ} \mathrm{C}$ corner. Similarly, we also find the $\mathrm{C}_{\mathrm{FLY}}$ requirement if the converter is at the TT and $25^{\circ} \mathrm{C}$ corner in Fig. 8(b) and at the FF and $80^{\circ} \mathrm{C}$ corner in Fig. 8(c). The largest CFLY across the three corners is $0.14 \mathrm{nF}$. This is $15 \times$ smaller than the $\mathrm{C}_{\mathrm{FLY}}$ requirement $(2.15 \mathrm{nF}$, Fig. 3(d)) found by the conventional worst-case methodology. Note that although the $\mathrm{V}_{\mathrm{DD}}$ needed for target throughput is very low for some cases (e.g., $\mathrm{FF}, 80^{\circ} \mathrm{C}$ ), the output voltage of the SC converter turns out to be higher than that to achieve the target PCE.

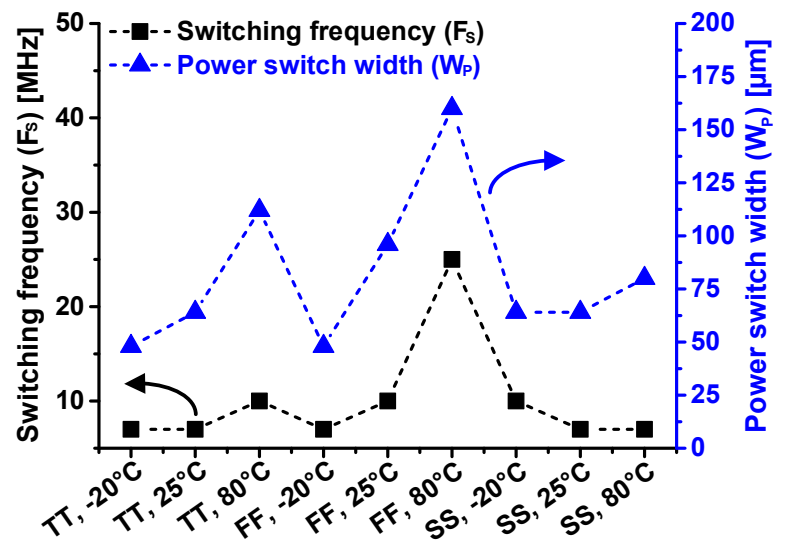

Fig. 9. Optimal $F_{S}$ and $W_{P}$ found by the proposed BTWC design methodology across process and temperature corners

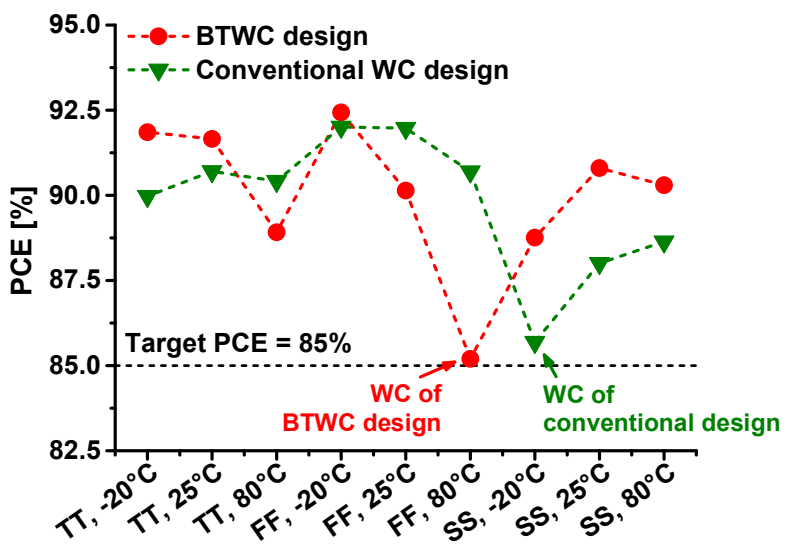

Fig. 10. PCEs of SC converters designed by the BTWC and the conventional WC design methodology
Finally, we consider the capacitor density variation together with other variations to set AFLY. Fig. 8(d) shows the MOS capacitor density at the SS and $-20^{\circ} \mathrm{C}$ corner considering the $\mathrm{V}_{D D}$ requirement. Since the load is in the same corner for the integrated scenario, the data points other than the leftmost are unrealistic. Similarly, in Figs. 8(e, f), we simulated AfLY for the two other corners. The largest AfLY across the three corners, which we need to commit, is $0.027 \mathrm{~mm}^{2}$. The AfLY determined by the worst-case methodology is $0.45 \mathrm{~mm}^{2}$ (Fig. 3(e)), confirming our proposed BTWC design methodology can scale the AFLY by $16.7 \times$.

Fig. 9 shows the $W_{P}$ and $F_{S}$ configurations that are found/used by the proposed BTWC methodology, with AFLY being optimized to $0.027 \mathrm{~mm}^{2}$. Frequency and conductance modulation techniques have been available and widely used $[11,12]$. With the configurations, the converter can achieve $>85 \%$ PCE across process and temperature corners. In general, as ILD increases, so do the optimal W. In addition, Fig. 10 shows the PCEs across the corners, achieved by the proposed BTWC design methodology. Again, the BTWC methodology yields AFLY $=0.027 \mathrm{~mm}^{2}$. The lowest $\mathrm{PCE}$ is achieved at the $\mathrm{FF}$ and $80^{\circ} \mathrm{C}$ corner. Fig. 10 also shows the PCEs of the converter designed by the conventional worst-case methodology. It shows similar PCEs across corners, but the smallest PCE appears at the SS and $-20^{\circ} \mathrm{C}$ corner. This is because the power switch resistance increases at this corner and degrades PCE.

\section{Impact of Load Current, Load $V_{D D}$, and On-chip Capacitor Type}

\subsection{Load Current Draw}

In this subsection, we experiment the scalability of the proposed design methodology. Particularly, the experiment in Sec. 3 uses a single arbitrary-chosen load current level $(0.18 \mathrm{~mA}$ at TT and $25^{\circ} \mathrm{C}$ ). Here we will verify the efficacy of the proposed methodology for a $125 \times$ range of load current from $0.04 \mu \mathrm{A}$ to 4.56 $\mathrm{mA}$.

Fig. 11(a) summarizes the AfLY results achieved by the proposed and the conventional design methodology. It shows that the AFLY increases roughly linearly with the load current in both methodologies. Fig. 11(b) summarizes the optimal $W_{P}$ and Fs across the ILD levels. In general, the optimal WP increase linearly with ILD while the optimal $F_{S}$ appear around $30 \mathrm{MHz}$ across the load current range considered. This experiment confirms that the proposed methodology maintains the benefits over a wide range of ILD levels.

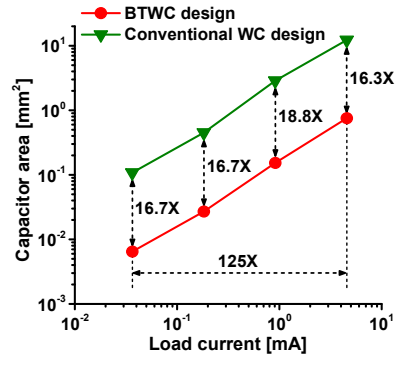

(a)

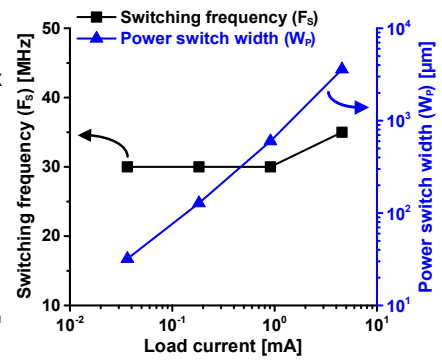

(b)
Fig. 11. (a) Capacitor area achieved by the BTWC and the conventional WC design methodology and (b) optimal Fs and $W_{P}$ found via the BTWC design methodology 


\subsection{Load VDD}

In this section, we investigate the impact of load $V_{D D}$ on the efficacy of the proposed BTWC methodology. The $\mathrm{V}_{\mathrm{DD}}$ of the load circuit considered in Sec. 3 is $0.24 \mathrm{~V}$ to $0.45 \mathrm{~V}$ across the process and temperature corners. In such near- and sub-threshold voltage regime, leakage takes a considerable amount of total current draw even in the TT and $25^{\circ} \mathrm{C}$ corner [13]. At the FF and $80^{\circ} \mathrm{C}$ corner, this leakage current increases exponentially, which is the root cause of the large variation of current draw across corners that we observed in Fig. 1.

At the load VDD that is higher than the threshold voltage, called super-threshold regime hereafter, leakage (static) current makes less impact on the total current draw. This makes the conventional worst-case design methodology less pessimistic for a superthreshold voltage load, as compared to near- and sub-threshold loads.

The aforementioned changes affect the savings from the BTWC methodology. Fig. 12 shows the results of our experiment, where we modulate the $\mathrm{V}_{\text {IN }}$ of the converter (Fig. 2) from 0.8 to $1.8 \mathrm{~V}$. The nominal output of the converter is $0.4 \mathrm{~V}$ to $0.9 \mathrm{~V}$. At $0.8 \mathrm{~V} \mathrm{~V}$ IN, we find that the proposed BTWC methodology can provide greater

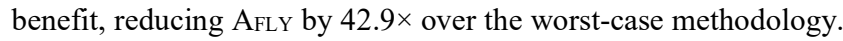
On the other hand, at the higher $\mathrm{V}_{\mathrm{IN}}$ of $1.8 \mathrm{~V}$, the BTWC methodology achieves a still considerable but less amount of improvement: $4.1 \times$ AfLY scaling as compared to the worst-case methodology. This is mainly because the ILD variability is smaller and thus the worst-case methodology becomes less pessimistic.

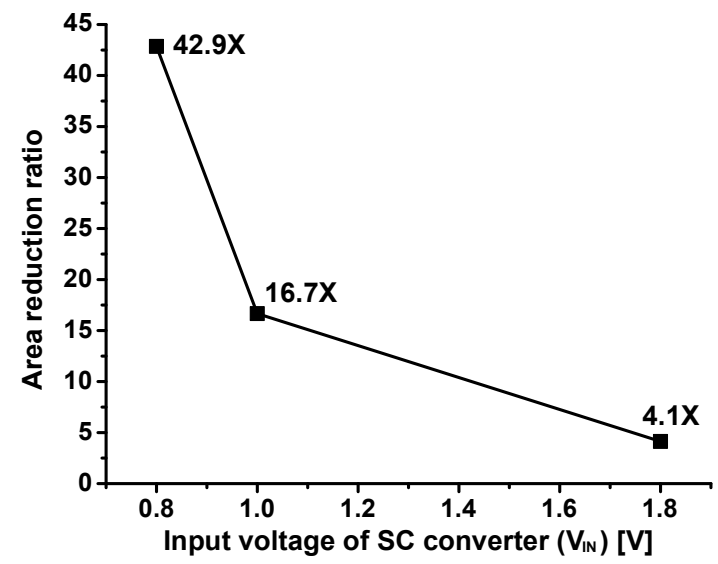

Fig. 12. Capacitor area scaling of $\mathrm{SC}$ converters designed by the BTWC and the conventional WC design methodology

\subsection{Capacitor Type}

In Sec. 3, we have considered the MOS capacitor to implement flying capacitors. In this subsection, we will consider the other popular type of the capacitor, metal-insulator-metal (MIM) capacitor, and investigate its impact on the proposed BTWC methodology. As compared to the MOS capacitor, the MIM capacitor typically has a better linearity over temperature and voltage variation. Also, it is implemented in the back end of CMOS technology, making it would not share process variation with transistors. Therefore, we have to consider the worst-case capacitance density to ensure the proper amount of charge transfer. We did the same benchmark converter design using MIM capacitors. The resulting AFLY savings over the worst-case methodology is $15 \times$, which is slightly lower than the savings achieved with MOS capacitors.

\section{Conclusion}

In this paper, we propose the BTWC design methodology for compact integrated SC DC-DC converters. The methodology aims to replace multiple worst-case assumptions with realistic and practical ones. Across ranges of load current and load $V_{D D}$, the proposed methodology successfully scales the size of a flying capacitor, an area-dominant component of an SC converter, by 4 to $43 \times$, while achieving the target PCE and the target throughput.

\section{Acknowledgement}

This work is supported by Semiconductor Research Corporation (SRC, Task 2712.012), NSF (CCF-1453142), and Catalyst Foundation. We appreciate Drs. Minki Cho, Ram Krishnamurthy, Seongjong Kim, Suhwan Kim and Suyoung Bang from Intel Circuit Research Lab (CRL) for valuable feedback and discussions.

\section{References}

[1] H.-P. Le, S. Sanders and E. Alon, "Design Techniques for Fully Integrated Switched-Capacitor DC-DC Converters," IEEE J. Solid-State Circuits, vol. 46, no. 9, pp. 2120-2131, Sep. 2011.

[2] S. T. Kim et al., "Enabling Wide Autonomous DVFS in a $22 \mathrm{~nm}$ Graphics Execution Core Using a Digitally Controlled Fully Integrated Voltage Regulator," IEEE J. Solid-State Circuits, vol. 51, no.1, pp. 18-30, Jan. 2016.

[3] S. Bang, D. Blaauw and D. Sylvester, "A Successive-Approximation Switched-Capacitor DC-DC Converter With Resolution of $\mathrm{V}_{\mathrm{IN}} / 2^{\mathrm{N}}$ for a Wide Range of Input and Output Voltages," IEEE J. Solid-State Circuits, vol. 51, no. 2, pp. 543-556, Feb. 2016.

[4] T. Austin et al., "Opportunities and challenges for better than worst-case design," in Proc. Asia and South Pacific Design Automation Conference (ASP-DAC), pp. I2-I7, Jan. 2005.

[5] M. Seok, D. Sylvester and D. Blaauw, "Optimal Technology Selection for Minimizing Energy and Variability in Low Voltage Applications," ACM/IEEE International Symposium on Low Power Electronics and Design (ISLPED), pp. 9-14, Aug. 2008

[6] A. Saiz-Vela et al., "Low-Power High-Voltage Non-overlapping Clock Generators for Switched-Capacitor step-up DC-DC Converters," IEEE International Midwest Symposium on Circuits and Systems (MWSCAS), pp. 61-64, Aug. 2006

[7] L. Chang et al., "A Fully-Integrated Switched-Capacitor 2:1 Voltage Converter with Regulation Capability and $90 \%$ Efficiency at $2.3 \mathrm{~A} / \mathrm{mm}^{2}$," IEEE Symposium on VLSI Circuits (VLSI), pp. 55-56, Jun. 2010.

[8] M. Wirnshofer, "Sources of Variation. In: Variation-Aware Adaptive Voltage Scaling for Digital CMOS Circuits," Springer Series in Advanced Microelectronics, vol. 41, Springer, Dordrecht.

[9] M. D. Seeman and S. R. Sanders, "Analysis and Optimization of Switched Capacitor DC-DC Converters," IEEE Trans. Power Electron., vol. 23, no. 2, pp. 841-851, Mar. 2008.

[10] M. Seok, "Decoupling capacitor design strategy for minimizing supply noise of ultra low voltage circuits," ACM/IEEE/EDAC Design Automation Conference (DAC), pp. 968-973, Jun. 2012.

[11] R. Jain et al., "Conductance Modulation Techniques in SwitchedCapacitor DC-DC Converter for Maximum-Efficiency Tracking and Ripple Mitigation in $22 \mathrm{~nm}$ Tri-Gate CMOS," IEEE J. Solid-State Circuits, vol. 50, no. 8, pp. 1809-1819, Aug. 2015.

[12] X. Mi et al., "Fully-Integrated Switched-Capacitor Voltage Regulator with On-Chip Current-Sensing and Workload Optimization in $32 \mathrm{~nm}$ SOI CMOS," ACM/IEEE International Symposium on Low Power Electronics and Design (ISLPED), pp. 140-145, Jul. 2015.

[13] Joao Pedro Cerqueira and Mingoo Seok, "Temporarily Fine-Grained Sleep Technique for Near- and Subthreshold Parallel Architectures," IEEE Trans. Very Large Scale Integration (VLSI) Systems, vol. 25, no. 1, pp. 189-197, Jan. 2017 